\section{Monitoring the monitors for anaesthesia}

\section{Joanne Michou}

\section{IMPACT OF MONITORING}

Monitoring during anaesthesia is paramount to patient safety and consists of hands-on techniques, such as pulse palpation, and the use of monitors that give us information that we could not obtain using our senses alone, such as end-tidal carbon dioxide $\left(\mathrm{EtCO}_{2}\right)$ from capnography. Monitors supplement clinical observation; they are not a replacement for the presence of an appropriately trained and experienced veterinary surgeon or veterinary nurse. An appropriately trained and experienced anaesthetist has been shown to be the main determinant of patient safety in human anaesthesia.

However, accidents, incidents and near misses occur with substantial evidence showing this is frequently attributable to human error. Monitors reduce the risk of incidents and accidents by both detecting the consequences of the error and providing early warning signs of deterioration in patient condition, if the anaesthetist has the ability to analyse and interpret the information correctly.

In veterinary anaesthesia, use of pulse monitoring and pulse oximetry was shown to have a 3-4-fold reduction in the odds of death in cats. In human anaesthesia, analysis of reported perioperative events suggests that a combination of capnography, pulse oximetry and arterial blood pressure monitoring would have allowed earlier detection of $93 \%$ of incidents.

Guidelines for minimum monitoring standards exist in both human and veterinary anaesthesia to improve care and safety.

\section{MONITORS AND MAINTAINING PHYSIOLOGICAL NORMALITY}

General anaesthesia reversibly renders a patient unconscious, muscle relaxed and provides analgesia, in a controlled manner. The drugs cause significant dose-dependent changes to cardiovascular, respiratory and neurological systems, amongst others. We attempt to maintain these systems as close to physiologically normal as possible to prevent irreversible cell damage, by ensuring adequate oxygen delivery to tissues, whilst ensuring the patient is not 'too light', preventing movement and ensuring no memory of the event, under different levels of procedural stimulation. Monitoring is a continuous process and must continue until the patient has fully recovered from the effects of the anaesthetic. We need to use all the information available to us, know normal parameter ranges for a patient, breed or species and each patient's baseline prior to anaesthesia, in order to be able to interpret the information from the monitor in conjunction with clinical observation.

It is difficult to practically measure some parameters, such as oxygen delivery to tissues, therefore we use a combination of parameters that we can assess.

\section{The cardiovascular system}

- Clinical observations:

- Heart rate (HR), rhythm

- Pulse palpation

- Mucous membrane colour

- Capillary refill time

- Temperature

- Monitoring device:

- Arterial blood pressure (ABP)

- Pulse oximetry

- Capnograph

- Electrocardiogram (ECG)

- Advanced monitoring:

- Urine output

- Arterial blood gas analysis

- Cardiac output monitoring.

\section{Respiratory system}

- Clinical observations:

- Respiratory rate (RR), effort, pattern, depth

- Mucous membrane colour

- Auscultation

- Monitoring device:

- Capnograph

- Pulse oximetry

- Advanced monitoring:

- Respiratory gas analysis

- Arterial blood gas analysis

- Spirometry.

\section{Neurological system and depth of anaesthesia}

- Clinical observations:

- Consciousness

- Reflexes - palpebral, globe position, gag

- Muscle tone

- Autonomic nervous system - HR, RR

- Monitoring device:

- Autonomic nervous system; HR, RR, ABP, EtCO

- Anaesthetic gas analysis

- Advanced monitoring:

- Electroencephalogram-based (EEG).

\section{KEY LEARNING OBJECTIVES}

- Understand about safety and monitoring

- Be aware of the importance of clinical observations

- Appreciate why we need monitors 


\section{MULTIPLE CHOICE QUESTIONS}

1. What has pulse oximetry monitoring in cats been shown to do?

(A) Reduce the odds of death

(B) Not be important

(C) Be unreliable

(D) Increase the odds of death

2. Which one does ECG NOT give us information on?

(A) Heart rate

(B) Electrical activity of the heart
(C) Cardiac output

(D) Cardiac rhythm

3. Effectively monitoring a patient that is anaesthetized consists of which of the following?

(A) Monitoring, including capnography, pulse oximetry and arterial blood pressure

(B) The presence of a suitably trained and experienced anaesthetist

(C) Clinical observations of the patient

(D) All of the above

\section{ECG monitoring under anaesthesia}

\section{Frances Downing}

In recent years, the use of an ECG has become commonplace in companion animal practice. An ECG provides us with information about the electrical activity of the heart, allowing determination of both the rhythm and heart rate. However, it does not tell us whether the heart is contracting normally and therefore should be used in conjunction with peripheral pulse palpation; ideally echocardiography should also be used when assessing cardiac function.

The use of an ECG during routine monitoring of patients during sedation and general anaesthesia can help identify cardiac rhythm disturbances. Whilst a large proportion of the arrhythmias seen during anaesthesia are benign and require no intervention, it is important to identify those that are haemodynamically and clinically significant. If untreated, these malignant arrhythmias can result in a reduction in cardiac output, arterial hypotension, hypoperfusion of tissues and even death.

Cardiac arrhythmias are an important cause of perioperative complications encountered in companion animal species. The causes of arrhythmias seen during anaesthesia can be broadly divided into three categories:

- Anaesthetic-related, including drug administration,

hypoxaemia, hypotension and hypothermia

- Procedural, including surgical stimulation and

haemorrhage

- Patient-related, including underlying cardiac disease and pre-existing systemic illness.

Successful management of cardiac arrhythmias under anaesthesia requires accurate identification of the nature of the arrhythmia itself, establishing and, where possible, correcting any factors that may have contributed to its development and selecting appropriate pharmacological interventions.

\section{KEY LEARNING OBJECTIVES}

- Develop confidence in identifying abnormal electrocardiogram (ECG) rhythms that may be encountered in patients following sedation and under general anaesthesia

- Understand when arrhythmias encountered under anaesthesia require intervention and which treatment options may be most appropriate

- Appreciate the arrhythmogenic potential of commonly used anaesthetic drugs

\section{MULTIPLE CHOICE QUESTIONS}

1. What does the ' $T$ ' wave on a normal ECG trace represent?
(A) Atrial depolarization
(B) Atrial repolarization
(C) Ventricular depolarization
(D) Ventricular repolarization

2. Which arrhythmia is depicted in the ECG trace in Figure 1?

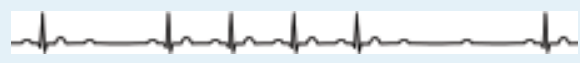

Figure 1: ECG trace.

(A) Sinus tachycardia

(B) Second-degree degree atrioventricular block

(C) Accelerated idioventricular rhythm

(D) Atrial fibrillation

3. The cardiac effects of medetomidine can be described as biphasic. Which of the following describes the phase 1 response?

(A) Peripheral vasoconstriction, hypertension and reflex bradycardia

(B) Peripheral vasoconstriction, hypertension and reflex tachycardia

(C) Peripheral vasodilation, hypotension and reflex bradycardia

(D) Peripheral vasodilation, hypotension and reflex tachycardia 